\title{
BEST APPROXIMATION AND QUASITRIANGULAR ALGEBRAS ${ }^{1}$ \\ BY \\ TIMOTHY G. FEEMAN
}

\begin{abstract}
If $\mathscr{P}$ is a linearly ordered set of projections on a Hilbert space and $\mathscr{K}$ is the ideal of compact operators, then $\mathrm{Alg} \mathscr{P}+\mathscr{K}$ is the quasitriangular algebra associated with $\mathscr{P}$. We study the problem of finding best approximants in a given quasitriangular algebra to a given operator: given $T$ and $\mathscr{P}$, is there an $A$ in Alg $\mathscr{P}+\mathscr{K}$ such that $\|T-A\|=\inf \{\|T-S\|: S \in \operatorname{Alg} \mathscr{P}+\mathscr{K}\}$ ? We prove that if $\mathscr{A}$ is an operator subalgebra which is closed in the weak operator topology and satisfies a certain condition $\Delta$, then every operator $T$ has a best approximant in $\mathscr{A}+\mathscr{K}$. We also show that if $\mathscr{E}$ is an increasing sequence of finite rank projections converging strongly to the identity then $\mathrm{Alg} \mathscr{E}$ satisfies the condition $\Delta$. Also, we show that if $T$ is not in $\mathrm{Alg} \mathscr{E}+\mathscr{K}$ then the best approximants in $\mathrm{Alg} \mathscr{E}+\mathscr{K}$ to $T$ are never unique.
\end{abstract}

1. Introduction. The concept of quasitriangular operators on a Hilbert space was introduced by Halmos in [5], where an operator $T$ is said to be quasitriangular if there is a sequence $\left\{E_{n}\right\}$ of finite rank projections strongly converging to the identity such that $\left\|\left(1-E_{n}\right) T E_{n}\right\| \rightarrow 0$.

For a fixed increasing sequence $\left\{P_{n}\right\}$ of finite rank projections strongly converging to the identity, Arveson [2] defined the quasitriangular algebra $Q T\left(\left\{P_{n}\right\}\right)$ to be the set of all operators $T$ for which $\left\|\left(1-P_{n}\right) T P_{n}\right\| \rightarrow 0$. He proved a distance formula for $Q T\left(\left\{P_{n}\right\}\right)$ and showed that $Q T\left(\left\{P_{n}\right\}\right)=\operatorname{Alg}\left\{P_{n}\right\}+\mathscr{K}$, where $\operatorname{Alg}\left\{P_{n}\right\}$ $=\left\{T:\left(1-P_{n}\right) T P_{n}=0\right.$ for all $\left.n\right\}$ is the triangular algebra associated with $\left\{P_{n}\right\}$ and $\mathscr{K}$ is the ideal of compact operators.

For any linearly ordered set $\mathscr{P}$ of projections which is closed in the strong operator topology and contains 0 and 1, Fall, Arveson, and Muhly [4] showed that the algebra Alg $\mathscr{P}+\mathscr{K}$ is norm closed, where $\mathrm{Alg} \mathscr{P}$ is the triangular algebra associated with $\mathscr{P}$, namely $\operatorname{Alg} \mathscr{P}=\{T:(1-P) T P=0$, all $P \in \mathscr{P}\}$. They also gave a characterization of Alg $\mathscr{P}+\mathscr{K}$ as a generalized quasitriangular algebra.

In this paper we study the problem of finding best quasitriangular approximants to a given operator: given an operator $T$ does there exist an operator $A$ in $\operatorname{Alg} \mathscr{P}+\mathscr{K}$ for which $\|T-A\|=\inf \{\|T-S\|: S \in \operatorname{Alg} \mathscr{P}+\mathscr{K}\}$ ? We prove that if $\mathscr{A}$ is an operator subalgebra which is closed in the weak operator topology and satisfies a certain condition $\Delta(\mathscr{A})$, then every operator $T$ has a best approximant in $\mathscr{A}+\mathscr{K}$.

Received by the editors March 13, 1984

1980 Mathematics Subject Classification. Primary 47D25; Secondary 41A50, 41A52.

Key words and phrases. Quasitriangular operator algebras, nest algebra, best approximation.

${ }^{1}$ This work represents a part of the author's doctoral dissertation at the University of Michigan. The author would like to thank Allen Shields for his guidance. 
We also show that if $\left\{P_{n}\right\}$ is an increasing sequence of finite rank projections strongly converging to 1 , then $\operatorname{Alg}\left\{P_{n}\right\}$ satisfies the condition $\Delta\left(\operatorname{Alg}\left\{P_{n}\right\}\right)$. Hence, best approximants in $\operatorname{Alg}\left\{P_{n}\right\}+\mathscr{K}$ exist for every operator $T$. Moreover, we show that if $T \notin \operatorname{Alg}\left\{P_{n}\right\}+\mathscr{K}$, then such best approximants are never unique.

Some of our results are reminiscent of those proved in [3] by Axler, Berg, Jewell, and Shields, where it is shown, for example, that every $L^{\infty}$ function on the unit circle has a best approximant in the algebra $H^{\infty}+C$. In fact, the general approach to proving our main result is inspired by that paper.

2. Preliminaries. In what follows, $H$ will be a separable infinite-dimensional Hilbert space with $\mathscr{L}(H)$ denoting the algebra of all bounded linear operators on $H$ and $\mathscr{K}(H)$, or simply $\mathscr{K}$, denoting the ideal of compact operators in $\mathscr{L}(H)$. All subspaces of $H$ are assumed to be closed and all projections are selfadjoint. For a projection $P$ let $P^{\perp}=1-P$.

If $\mathscr{S}$ is any subset of $\mathscr{L}(H)$ and $T \in \mathscr{L}(H)$, then the distance of $T$ from $\mathscr{S}$ is given by $d(T, \mathscr{S})=\inf \{\|T-S\|: S \in \mathscr{S}\}$. Also, Lat $\mathscr{S}$ will denote the set of all projections $P$ for which $P S P=S P$ whenever $S \in \mathscr{S}$. If $\mathscr{P}$ is a set of projections in $\mathscr{L}(H)$, then Alg $\mathscr{P}$ denotes the set of all operators $T$ in $\mathscr{L}(H)$ for which $P T P=T P$ whenever $P \in \mathscr{P}$. A subalgebra $\mathscr{S} \subset \mathscr{L}(H)$ is said to be reflexive if $\mathrm{Alg}$ Lat $\mathscr{S}=\mathscr{S}$.

A nest is a family of projections which is linearly ordered by range inclusion, contains 0 and 1 , and is closed in the strong operator topology (SOT). A nest algebra is a subalgebra $\mathscr{A}$ of $\mathscr{L}(H)$ for which $\mathscr{A}=$ Alg $\mathscr{P}$ for some nest $\mathscr{P}$. Equivalently, it is not hard to see that a nest algebra is a reflexive algebra $\mathscr{A}$ such that Lat $\mathscr{A}$ is linearly ordered (cf. [9]).

In [2] Arveson established the following distance formula for a nest algebra $\mathscr{A}$.

$$
d(T, \mathscr{A})=\sup \left\{\left\|P^{\perp} T P\right\|: P \in \text { Lat } \mathscr{A}\right\} \quad \text { for } T \in \mathscr{L}(H) .
$$

For a nest $\mathscr{P}$ define the quasitriangular algebra associated with $\mathscr{P}$ by $Q T(\mathscr{P})=$ Alg $\mathscr{P}+\mathscr{K}(H)$. In [4] Fall, Arveson, and Muhly showed that $Q T(\mathscr{P})$ is a norm closed algebra and that

$$
\begin{array}{r}
Q T(\mathscr{P})=\left\{T \in \mathscr{L}(H): \text { (i) } P^{\perp} T P \in \mathscr{K}(H), \text { for all } P \in \mathscr{P},\right. \\
\text { the map } P \mapsto P^{\perp} T P \text { is continuous } \\
\text { (ii) with respect to the SOT on } \mathscr{P} \text { and } \\
\text { the norm topology on } \mathscr{K}(H)\} .
\end{array}
$$

In the case when $\mathscr{P}=\left\{P_{n}\right\}$ is an increasing sequence of finite rank projections converging strongly to 1 , this yields the definition of $Q T\left(\left\{P_{n}\right\}\right)$ given by Arveson in [2]. For this special case Arveson has established the following distance formula.

$$
d\left(T, Q T\left(\left\{P_{n}\right\}\right)\right)=\varlimsup_{\lim }\left\|P_{n}^{\perp} T P_{n}\right\|, \quad n \rightarrow \infty, \text { for } T \in \mathscr{L}(H) .
$$

In this case (2.1) can be written as

$$
d\left(T, \operatorname{Alg}\left\{P_{n}\right\}\right)=\sup \left\{\left\|P_{n}^{\perp} T P_{n}\right\|: \text { all } n\right\} \quad \text { for } T \in \mathscr{L}(H) .
$$

We also need the following known result. 
LeMma 2.3. If $\mathscr{A} \subset \mathscr{L}(H)$ is closed in the weak operator topology (WOT), then every $T$ in $\mathscr{L}(H)$ has a best approximant in $\mathscr{A}$.

Proof. The proof is a standard argument using the compactness, in the weak operator topology, of the closed unit ball in $\mathscr{L}(H)$.

Finally, we observe that if $\mathscr{P}$ is a nest then Alg $\mathscr{P}$ is closed in the WOT. Indeed, if $\left\{A_{\lambda}\right\} \subset \mathrm{Alg} \mathscr{P}$ is a net of operators such that $A_{\lambda} \rightarrow A$ (WOT), then, for each $P \in \mathscr{P}$, $0=P^{\perp} A_{\lambda} P \rightarrow P^{\perp} A P$ (WOT), which implies that $A \in \mathrm{Alg} \mathscr{P}$.

\section{Main results.}

DeFINITION 3.1. A subalgebra $\mathscr{A}$ of $\mathscr{L}(H)$ satisfies condition $\Delta(\mathscr{A})$ provided that, for each $T \in \mathscr{L}(H)$, for each sequence of operators $\left\{A_{n}\right\} \subset \mathscr{L}(H)$ satisfying $A_{n} \rightarrow 0$ (SOT), and for each $\varepsilon>0$, there exists an $N$ such that

$$
d\left(T+A_{N}, \mathscr{A}\right) \leqslant \varepsilon+\max \left\{d(T, \mathscr{A}), d(T, \mathscr{A}+\mathscr{K})+d\left(A_{N}, \mathscr{A}\right)\right\} .
$$

Two remarks are in order. First, if condition $\Delta(\mathscr{A})$ holds and $T,\left\{A_{n}\right\}$, and $\varepsilon$ are chosen as indicated, then there exists an $N$ such that

$$
d\left(T+\beta A_{N}, \mathscr{A}\right) \leqslant \varepsilon+\max \left\{d(T, \mathscr{A}), d(T, \mathscr{A}+\mathscr{K})+d\left(\beta A_{N}, \mathscr{A}\right)\right\}
$$

for all $\beta \in[0,1]$. Otherwise, for each $n$, take $\beta_{n} \in[0,1]$ such that the inequality fails for $\beta_{n} A_{n}$. The sequence $\left\{\beta_{n} A_{n}\right\}$ satisfies $\beta_{n} A_{n} \rightarrow 0$ (SOT), so the assumption that condition $\Delta(\mathscr{A})$ holds yields a contradiction. Secondly, for any fixed $M, N$ can be chosen so that $N \geqslant M$ by restricting attention to the sequence $\left\{A_{n}: n \geqslant M\right\}$.

The next result enables us to reduce the problem of finding best approximants in $\mathscr{A}+\mathscr{K}(H)$ to that of finding best approximants in $\mathscr{A}$.

THEOREM 3.2. Let $\mathscr{A} \subset \mathscr{L}(H)$ be a subalgebra satisfying condition $\Delta(\mathscr{A})$. Choose $T \in \mathscr{L}(H) \backslash \mathscr{A}+\mathscr{K}$ and suppose the sequence $\left\{T_{n}\right\} \subset \mathscr{A}+\mathscr{K}$ satisfies $T_{n} \rightarrow T(S O T)$. Then there is a sequence $\left\{a_{n}\right\}$ of nonnegative real numbers satisfying $\sum a_{n}=1$ and such that, if $K=\sum a_{n} T_{n}$, then $d(T-K, \mathscr{A})=d(T, \mathscr{A}+\mathscr{K})$.

Proof. Let $A_{n}=T-T_{n}$ so that $A_{n} \rightarrow 0$ (SOT). For convenience let

$$
r=d(T, \mathscr{A}+\mathscr{K}) .
$$

Claim. There exists an increasing sequence of positive integers $\{n(k)\}$ and a sequence $\left\{\alpha_{k}\right\}$ of positive real numbers such that $\sum \alpha_{k}=1$ and such that, for all $N=1,2, \ldots$,

$$
d\left(\sum_{k=1}^{N} \alpha_{k} A_{n(k)}, \mathscr{A}\right)=r-\varepsilon_{N}, \quad \text { where } \varepsilon_{N}=r / 3^{N} .
$$

Proof of Claim. Choose $n(1)=1$. Since $A_{1} \notin \mathscr{A}+\mathscr{K}$, it follows that $d\left(A_{1}, \mathscr{A}\right)$ $\neq 0$. Choose $\alpha_{1}$ such that $\alpha_{1} \cdot d\left(A_{1}, \mathscr{A}\right)=r-\varepsilon_{1}$. Since $\alpha_{1} \cdot d\left(A_{1}, \mathscr{A}\right)=$ $d\left(\alpha_{1} A_{1}, \mathscr{A}\right)$, it follows that $d\left(\alpha_{1} A_{1}, \mathscr{A}\right)=r-\varepsilon_{1}$. The relations

$$
d\left(A_{1}, \mathscr{A}\right)=d\left(T, \mathscr{A}+T_{1}\right) \geqslant d(T, \mathscr{A}+\mathscr{K})
$$

imply $0<\alpha_{1}<1$.

Suppose $n(1), \ldots, n(N)$ and $\alpha_{1}, \ldots, \alpha_{N}$ have been chosen as required. Applying condition $\Delta(\mathscr{A})$ to the operator $\sum_{k=1}^{N} \alpha_{k} A_{n(k)}$, the sequence $\left\{A_{n}\right\}$, and $\varepsilon_{N+1}$, choose 
$n(N+1)>n(N)$ such that

$$
\begin{array}{r}
d\left(\sum_{k=1}^{N} \alpha_{k} A_{n(k)}+\beta A_{n(N+1)}, \mathscr{A}\right) \\
\leqslant \varepsilon_{N+1}+\max \left\{d\left(\sum_{k=1}^{N} \alpha_{k} A_{n(k)}, \mathscr{A}\right), d\left(\sum_{k=1}^{N} \alpha_{k} A_{n(k)}, \mathscr{A}+\mathscr{K}\right)\right. \\
\left.+d\left(\beta A_{n(N+1)}, \mathscr{A}\right)\right\} \\
\quad \text { for all } \beta \in[0,1] .
\end{array}
$$

Consider the quantity $d\left(\sum_{k=1}^{N} \alpha_{k} A_{n(k)}+\alpha A_{n(N+1)}, \mathscr{A}\right)$ as a function of $\alpha$. When $\alpha=0$ this quantity equals $r-\varepsilon_{N}$. Note that $r-\varepsilon_{N}<r-\varepsilon_{N+1}$. As $\alpha \rightarrow \infty$ this quantity also approaches $\infty$. (Here we use the fact that $A_{k}$ does not belong to $\mathscr{A}$ for any $k$.) Thus, there exists some value of $\alpha$, call it $\alpha_{N+1}$, for which

$$
d\left(\sum_{k=1}^{N} \alpha_{k} A_{n(k)}+\alpha_{N+1} \cdot A_{n(N+1)}, \mathscr{A}\right)=r-\varepsilon_{N+1} .
$$

Note that

$$
\begin{aligned}
r-\varepsilon_{N+1} & =d\left(\sum_{k=1}^{N+1} \alpha_{k} A_{n(k)}, \mathscr{A}\right)=d\left(\sum_{k=1}^{N+1} \alpha_{k}\left(T-T_{n(k)}\right), \mathscr{A}\right) \\
& \geqslant d\left(\sum_{k=1}^{N+1} \alpha_{k} T, \mathscr{A}+\mathscr{K}\right) \text { since } T_{n(k)} \in \mathscr{A}+\mathscr{K} \\
& =\left(\sum_{k=1}^{N+1} \alpha_{k}\right) \cdot d(T, \mathscr{A}+\mathscr{K})=\left(\sum_{k=1}^{N+1} \alpha_{k}\right) \cdot r
\end{aligned}
$$

and, hence, $\sum_{k=1}^{N+1} \alpha_{k}<1$. It remains to show that $\sum \alpha_{k}=1$.

Referring to inequality (3.3), with $\alpha_{N+1}$ in place of $\beta$, suppose that

$$
d\left(\sum_{k=1}^{N+1} \alpha_{k} A_{n(k)}, \mathscr{A}\right) \leqslant \varepsilon_{N+1}+d\left(\sum_{k=1}^{N} \alpha_{k} A_{n(k)}, \mathscr{A}\right) .
$$

Then $r-\varepsilon_{N+1} \leqslant \varepsilon_{N+1}+\left(r-\varepsilon_{N}\right)$, which implies that $\varepsilon_{N} \leqslant 2 \varepsilon_{N+1}$, a contradiction of the definition of $\left\{\varepsilon_{n}\right\}$. It follows that

$$
\begin{aligned}
r-\varepsilon_{N+1} & =d\left(\sum_{k=1}^{N+1} \alpha_{k} A_{n(k)}, \mathscr{A}\right) \\
& \leqslant \varepsilon_{N+1}+d\left(\sum_{k=1}^{N} \alpha_{k} A_{n(k)}, \mathscr{A}+\mathscr{K}\right)+d\left(\alpha_{N+1} A_{n(N+1)}, \mathscr{A}\right) \\
& =\varepsilon_{N+1}+\left(\sum_{k=1}^{N} \alpha_{k}\right) \cdot r+\alpha_{N+1} \cdot d\left(A_{n(N+1)}, \mathscr{A}\right) .
\end{aligned}
$$

If $N \rightarrow \infty$ then $\varepsilon_{N+1} \rightarrow 0$ and, since $\sum \alpha_{k} \leqslant 1$, it follows that $\alpha_{N+1} \rightarrow 0$. Since $A_{n} \rightarrow 0$ (SOT) we see that $\left\{\left\|A_{n}\right\|\right\}$, and hence $\left\{d\left(A_{n}, \mathscr{A}\right)\right\}$, is a bounded set. Thus, 
letting $N \rightarrow \infty$ in the above yields $r=d\left(\sum \alpha_{k} A_{n(k)}, \mathscr{A}\right) \leqslant\left(\sum \alpha_{k}\right) \cdot r$, which implies that $\sum \alpha_{k} \geqslant 1$. This completes the proof of the claim.

To complete the proof of the theorem, define the sequence $\left\{a_{n}\right\}$ by $a_{n(k)}=\alpha_{k}$ and $a_{j}=0$ if $j$ is not of the form $n(k)$ for any $k$. Also, let $K=\sum a_{n} T_{n}=\sum \alpha_{k} T_{n(k)}=T-$ $\sum \alpha_{k} A_{n(k)}$. This sum converges since $\sum \alpha_{k}=1$ and since $\left\{\left\|A_{n}\right\|\right\}$ is a bounded set. It follows from the foregoing discussion that $d(T-K, \mathscr{A})=d\left(\sum \alpha_{k} A_{n(k)}, \mathscr{A}\right)=r=$ $d(T, \mathscr{A}+\mathscr{K})$, which completes the proof.

Note that if $\mathscr{A}+\mathscr{K}(H)$ is norm closed then $K \in \mathscr{A}+\mathscr{K}(H)$. Also, if $\left\{T_{n}\right\}$ is taken to be a sequence of compact operators converging to $T$ (SOT), then $K \in \mathscr{K}(H)$, since $\mathscr{K}(H)$ is norm closed.

We are now in a position to prove one of our main results on the existence of best approximants.

THEOREM 3.4. Let $\mathscr{A} \subset \mathscr{L}(H)$ be a subalgebra which is WOT-closed and satisfies condition $\Delta(\mathscr{A})$, and suppose $T \in \mathscr{L}(H)$. Then there exists $B \in \mathscr{A}+\mathscr{K}(H)$ such that $\|T-B\|=d(T, \mathscr{A}+\mathscr{K}(H))$.

Proof. Assume $T \in \mathscr{L}(H) \backslash \mathscr{A}+\mathscr{K}$, since otherwise the result is obvious. Let $\left\{e_{j}: j \geqslant 0\right\}$ be an orthonormal basis for $H$ and define $E_{n}$ to be the projection onto the subspace spanned by $\left\{e_{j}: j \leqslant n\right\}$. Each $E_{n}$ has finite rank and $E_{n} \rightarrow 1$ (SOT). Set $T_{n}=E_{n} T E_{n}$. Each $T_{n}$ is compact and $T_{n} \rightarrow T(\mathrm{SOT})$.

By Theorem 3.2 there is a sequence $\left\{a_{n}\right\}$ of nonnegative real numbers satisfying $\sum a_{n}=1$ and such that, if $K=\sum a_{n} T_{n}, d(T-K, \mathscr{A})=d(T, \mathscr{A}+\mathscr{K})$. Note that $K \in \mathscr{K}$. By Lemma 2.3 there exists $A \in \mathscr{A}$ such that $\|(T-K)-A\|=d(T-K, \mathscr{A})$. Therefore, the operator $B=A+K$ is in $\mathscr{A}+\mathscr{K}$ and satisfies

$$
\|T-B\|=d(T, \mathscr{A}+\mathscr{K}) .
$$

In other words, $B$ is a best approximant to $T$ in $\mathscr{A}+\mathscr{K}$.

We remarked earlier that every nest algebra is WOT-closed, so Theorem 3.4 applies, in particular, to any nest algebra $\mathscr{A}$ which satisfies condition $\Delta(\mathscr{A})$.

The following corollary shows that if $\mathscr{A}+\mathscr{K}$ is norm closed, then the operator $K$ in the conclusion of Theorem 3.2 is not unique.

Corollary 3.5. Let $\mathscr{A}, T$, and $\left\{T_{n}\right\}$ be as in the statement of Theorem 3.2, and also suppose that $\mathscr{A}+\mathscr{K}$ is norm closed. Then there exist two sequences $\left\{a_{n}\right\}$ and $\left\{b_{n}\right\}$ of nonnegative real numbers such that $\sum a_{n}=\sum b_{n}=1$ and such that, if $K=\sum a_{n} T_{n}$ and $K_{1}=\sum b_{n} T_{n}$, then $K \neq K_{1}$ and $d(T-K, \mathscr{A})=d\left(T-K_{1}, \mathscr{A}\right)=d(T, \mathscr{A}+\mathscr{K})$.

Proof. Let $\left\{a_{n}\right\}$ and $K=\sum a_{n} T_{n}$ be as in the conclusion of Theorem 3.2. Then $\left(T_{n}-K\right) \rightarrow(T-K)$ (SOT). Let $\mathcal{O}$ be a convex neighborhood of $T-K$ in the strong operator topology whose closure does not contain $\mathcal{O}$. Deleting a finite number of terms if necessary, assume that $T_{n}-K \in \mathcal{O}$ for all $n$.

Since $\mathscr{A}+\mathscr{K}$ is norm closed, we see that $K \in \mathscr{A}+\mathscr{K}$ and, hence, $\left(T_{n}-K\right) \in \mathscr{A}+$ $\mathscr{K}$ for all $n$. Thus by Theorem 3.2 we can construct a sequence $\left\{b_{n}\right\}$ such that $\Sigma b_{n}=1$ and such that if $K^{\prime}=\sum b_{n}\left(T_{n}-K\right)$, then

$$
d\left((T-K)-K^{\prime}, \mathscr{A}\right)=d(T-K, \mathscr{A}+\mathscr{K})=d(T, \mathscr{A}+\mathscr{K}) .
$$


Thus, the operator $K_{1}=K+K^{\prime}$ satisfies $d\left(T-K_{1}, \mathscr{A}\right)=d(T, \mathscr{A}+\mathscr{K})$. Since $K^{\prime}$ is a convex combination of elements of $\mathcal{O}$, it follows that $K^{\prime} \neq 0$ and, hence, $K_{1} \neq K$. This proves the corollary.

We noted earlier that $\mathscr{A}+\mathscr{K}$ is norm closed whenever $\mathscr{A}$ is a nest algebra, so Corollary 3.5 applies, in particular, to any nest algebra satisfying condition $\Delta(\mathscr{A})$. Also note that if $\left\{T_{n}\right\}$ is taken to be a sequence of compact operators, then $K$ is compact as well and the requirement that $\mathscr{A}+\mathscr{K}$ be norm closed is superfluous.

4. More main results. Throughout this section let $\mathscr{P}=\left\{P_{n}\right\}$ be a fixed increasing sequence of finite rank projections such that $P_{n} \rightarrow 1$ (SOT). Let

and let

$$
\mathscr{A}=\operatorname{Alg}\left\{P_{n}\right\}=\left\{T \in \mathscr{L}(H): P_{n}^{\perp} T P_{n}=0 \text { for all } n\right\}
$$

$$
Q T=Q T\left(\left\{P_{n}\right\}\right)=\left\{T \in \mathscr{L}(H):\left\|P_{n}^{\perp} T P_{n}\right\| \rightarrow 0, n \rightarrow \infty\right\} .
$$

The following result establishes the validity of condition $\Delta(\mathscr{A})$ in this special case. It then follows from Theorem 3.4 that best approximants in $Q T$ exist for every operator in $\mathscr{L}(H)$.

Proposition 4.1. The algebra $\mathscr{A}=\operatorname{Alg}\left\{P_{n}\right\}$ satisfies condition $\Delta(\mathscr{A})$.

Proof. Choose $T \in \mathscr{L}(H)$ and let $\left\{A_{n}\right\} \subset \mathscr{L}(H)$ satisfy $A_{n} \rightarrow 0$ (SOT). Fix $\varepsilon>0$. If condition $\Delta(\mathscr{A})$ is not satisfied, then by the distance formulas (2.2) and $\left(2.1^{\prime}\right)$ there is a sequence $\left\{m_{n}\right\}$ of nonnegative integers such that $\left\|P_{m_{n}}^{\perp}\left(T+A_{n}\right) P_{m_{n}}\right\|$ $>\varepsilon+\alpha_{n}$, where

$$
\alpha_{n}=\max \left(\sup _{j \geqslant 0}\left\|P_{j}^{\perp} T P_{j}\right\|, \varlimsup_{k}\left\|P_{k}^{\perp} T P_{k}\right\|+\sup _{j \geqslant 0}\left\|P_{j}^{\perp} A_{n} P_{j}\right\|\right) .
$$

Consider two cases.

Case 1. Suppose no nonnegative integer appears infinitely often in the sequence $\left\{m_{n}\right\}$. Passing to a subsequence if necessary, assume that $\left\{m_{n}\right\}$ is an increasing sequence. By the definition of lim sup, there exists some $N$ such that $n \geqslant N$ implies that $\left\|P_{m_{n}}^{\perp} T P_{m_{n}}\right\| \leqslant \varlimsup_{\lim _{k}}\left\|P_{k}^{\perp} T P_{k}\right\|+\varepsilon / 2$. Thus, for $n \geqslant N$, we have

$$
\begin{aligned}
\left\|P_{m_{n}}^{\perp}\left(T+A_{n}\right) P_{m_{n}}\right\| & \leqslant\left\|P_{m_{n}}^{\perp} T P_{m_{n}}\right\|+\left\|P_{m_{n}}^{\perp} A_{n} P_{m_{n}}\right\| \\
& \leqslant \varlimsup_{k}\left\|P_{k}^{\perp} T P_{k}\right\|+\varepsilon / 2+\sup _{j}\left\|P_{j}^{\perp} A_{n} P_{j}\right\| \\
& \leqslant \alpha_{n}+\varepsilon / 2 .
\end{aligned}
$$

This contradicts the definition of the sequence $\left\{m_{n}\right\}$.

Case 2. Suppose some nonnegative integer, call it $M$, appears infinitely often in the sequence $\left\{m_{n}\right\}$. Passing to a subsequence if necessary, assume that

$$
\left\|P_{M}^{\perp}\left(T+A_{n}\right) P_{M}\right\|>\varepsilon+\alpha_{n} \text { for all } n \text {. }
$$

Since $P_{M}$ is compact and $A_{n} \rightarrow 0$ (SOT), it follows that $\left\|A_{n} P_{M}\right\| \rightarrow 0$. Choose $N$ such that $\left\|A_{N} P_{M}\right\|<\varepsilon / 2$. We then have

$$
\begin{aligned}
\left\|P_{M}^{\perp}\left(T+A_{N}\right) P_{M}\right\| & \leqslant\left\|P_{M}^{\perp} T P_{M}\right\|+\left\|P_{M}^{\perp} A_{N} P_{M}\right\| \\
& \leqslant \sup _{j}\left\|P_{j}^{\perp} T P_{j}\right\|+\varepsilon / 2 \leqslant \alpha_{N}+\varepsilon / 2 .
\end{aligned}
$$


This yields a contradiction to the definition of the sequence $\left\{m_{n}\right\}$ and completes the proof of the proposition.

We now show that best approximants in $Q T$ are never unique for operators not in $Q T$.

Proposition 4.2. For each $T \in \mathscr{L}(H) \backslash Q T$ there exist operators $B$ and $B_{1}$ in $Q T$ such that $B \neq B_{1}$ and $\|T-B\|=\left\|T-B_{1}\right\|=d(T, Q T)$.

Proof. Consider two cases.

Case 1. Suppose there is a subsequence $\left\{n_{k}\right\}$ such that $\left(P_{n_{k+1}}-P_{n_{k}}\right) T P_{n_{0}} \neq 0$ for all $k \geqslant 0$. Set $E_{k}=P_{n_{k}}$ and let $T_{k}=E_{k} T E_{k}$. Now, let $K=\sum a_{k} T_{k}$ and $K_{1}=\sum b_{k} T_{k}$ be as in the conclusion of Corollary 3.5. Note that $K$ and $K_{1}$ are compact. By Lemma 2.3 we can find operators $A$ and $A_{1}$ in $\mathscr{A}$ such that $\|T-K-A\|=$ $d(T-K, \mathscr{A})$ and $\left\|T-K_{1}-A_{1}\right\|=d\left(T-K_{1}, \mathscr{A}\right)$. Thus, $B=A+K$ and $B_{1}=A_{1}$ $+K_{1}$ are best approximants in $Q T$ to $T$. To show that $B \neq B_{1}$, it suffices to show that $K-K_{1} \notin \mathscr{A}$.

Suppose, to the contrary, that $K-K_{1} \in \mathscr{A}$. Then it follows, in particular, that

$$
\begin{aligned}
0 & =E_{0}^{\perp}\left(K-K_{1}\right) E_{0}=\sum_{k \geqslant 0}\left(a_{k}-b_{k}\right) E_{0}^{\perp} E_{k} T E_{k} E_{0} \\
& =\sum_{k \geqslant 1}\left(a_{k}-b_{k}\right)\left(E_{k}-E_{0}\right) T E_{0} .
\end{aligned}
$$

Letting $C_{k}=\sum_{j \geqslant k}\left(a_{j}-b_{j}\right)$, a summation by parts shows that

$$
\sum_{k=1}^{N} C_{k}\left(E_{k}-E_{k-1}\right) T E_{0}=\sum_{k=1}^{N-1}\left(a_{k}-b_{k}\right) E_{k} T E_{0}+C_{N} E_{N} T E_{0}-C_{1} E_{0} T E_{0}
$$

As $N \rightarrow \infty,\left|C_{N}\right| \rightarrow 0$, so $\left\|C_{N} E_{N} T E_{0}\right\| \rightarrow 0$. Thus,

$$
\sum_{k=1}^{\infty} C_{k}\left(E_{k}-E_{k-1}\right) T E_{0}=\sum_{k=1}^{\infty}\left(a_{k}-b_{k}\right) E_{k} T E_{0}-C_{1} E_{0} T E_{0}
$$

We thus have that

$$
\begin{aligned}
\sum_{k=1}^{\infty}\left(\sum_{j \geqslant k}\left(a_{j}-b_{j}\right)\right)\left(E_{k}-E_{k-1}\right) T E_{0} & \\
= & \sum_{k=1}^{\infty}\left(a_{k}-b_{k}\right) E_{k} T E_{0}-\sum_{k=1}^{\infty}\left(a_{k}-b_{k}\right) E_{0} T E_{0} \\
= & \sum_{k=1}^{\infty}\left(a_{k}-b_{k}\right)\left(E_{k}-E_{0}\right) T E_{0}=0
\end{aligned}
$$

Since the range of $\left(E_{l}-E_{l-1}\right)$ is orthogonal to that of $\left(E_{j}-E_{j-1}\right)$ whenever $l \neq j$, and since, by assumption, $\left(E_{k}-E_{k-1}\right) T E_{0} \neq 0$ for $k \geqslant 1$, it follows that

$$
\sum_{j \geqslant k}\left(a_{j}-b_{j}\right)=0 \text { for } k \geqslant 1 \text {. }
$$

The fact that $\sum a_{n}=\sum b_{n}=1$ implies that $\sum_{j \geqslant 0}\left(a_{j}-b_{j}\right)=0$ as well. Hence, $a_{j}=b_{j}$ for all $j \geqslant 0$, which contradicts the assumption that $K \neq K_{1}$. Thus, $K-K_{1} \notin \mathscr{A}$ and, consequently, $B \neq B_{1}$. 
Case 2. Suppose there is no subsequence $\left\{n_{k}\right\}$ for which $\left(P_{n_{k+1}}-P_{n_{k}}\right) T P_{n_{0}} \neq 0$ for all $k$. Then for each $k$ there is a smallest integer $m(k)$ such that $P_{m(k)}^{\perp} T P_{k}=0$. We claim that $m(k) \geqslant k+1$ for infinitely many $k$. Indeed, were this not so then there would exist $N$ such that $m(k) \leqslant k$ for $k \geqslant N$. Hence, $P_{k}^{\perp} T P_{k}=0$ for $k \geqslant N$, which implies that $d(T, Q T)=0$, contradicting the assumption that $T \notin Q T$.

We make the following remarks.

(a) If $m(k) \geqslant k+1$, then $\left(P_{m(k)}-P_{k}\right) T P_{k} \neq 0$. This follows from the choice of $m(k)$ as the smallest integer such that $P_{m(k)}^{\perp} T P_{k}=0$.

(b) It is clear that if $\left(P_{m(k)}-P_{k}\right) T P_{k} \neq 0$, then $\left(P_{j}-P_{k}\right) T P_{k} \neq 0$ for $j \geqslant m(k)$.

Now, choose $k_{0}$ such that $m\left(k_{0}\right) \geqslant k_{0}+1$ and $T P_{k_{0}} \neq 0$. For $j \geqslant 1$ inductively choose $k_{j}$ such that $m\left(k_{j}\right) \geqslant k_{j}+1$ and $k_{j}>m\left(k_{j-1}\right)$. Set $E_{j}=P_{k_{j}}$ and let $T_{j}=$ $E_{j} T E_{j}$. From this we get $K=\sum a_{n} T_{n}$ and $K_{1}=\sum b_{n} T_{n}$, as in the conclusion of Corollary 3.5. To complete the proof it suffices, as in the previous case, to show that $K-K_{1} \notin \mathscr{A}$.

First observe that remarks (a) and (b) imply that $\left(E_{n}-E_{l}\right) T E_{l} \neq 0$ for $n \geqslant l+1$. Also, by the construction of the sequence $\left\{E_{n}\right\}$, it follows that $\left(E_{j+1}-E_{j}\right) T E_{l}=0$ for $j \geqslant l+1$. Putting these together we see that, for $n \geqslant l+1$,

$$
\left(E_{n}-E_{l}\right) T E_{l}=\sum_{j=l}^{n-1}\left(E_{j+1}-E_{j}\right) T E_{l}=\left(E_{l+1}-E_{l}\right) T E_{l} \neq 0 .
$$

To see that $K-K_{1} \notin \mathscr{A}$, suppose the contrary. Then, for $l \geqslant 0$, we must have

$$
\begin{aligned}
0 & =E_{l}^{\perp}\left(K-K_{1}\right) E_{l}=\sum_{n \geqslant 0}\left(a_{n}-b_{n}\right) E_{l}^{\perp} E_{n} T E_{n} E_{l} \\
& =\sum_{n \geqslant l+1}\left(a_{n}-b_{n}\right)\left(E_{n}-E_{l}\right) T E_{l} \\
& =\sum_{n \geqslant l+1}\left(a_{n}-b_{n}\right)\left(E_{l+1}-E_{l}\right) T E_{l} \\
& =\left[\sum_{n \geqslant l+1}\left(a_{n}-b_{n}\right)\right]\left(E_{l+1}-E_{l}\right) T E_{l} .
\end{aligned}
$$

Since $\left(E_{l+1}-E_{l}\right) T E_{l} \neq 0$, it follows that $\sum_{n \geqslant l+1}\left(a_{n}-b_{n}\right)=0$ for $l \geqslant 0$. Since $\sum a_{n}=\sum b_{n}=1$, it follows that $\sum_{n \geqslant l}\left(a_{n}-b_{n}\right)=0$ for all $l \geqslant 0$ and, hence, $a_{n}=b_{n}$ for all $n$, contradicting the assumption that $K \neq K_{1}$. Hence, $K-K_{1} \notin \mathscr{A}$ and the corollary is proved.

5. Remarks. The obvious question is to ask which subalgebras $\mathscr{A}$ satisfy the condition $\Delta(\mathscr{A})$. Our proof of Proposition 4.1 and Arveson's proof of the distance formula (2.2) both use the finite dimensionality of the projections $P_{n}$ in an important way. Some means of eliminating this dependence would apparently be needed to establish a broader validity of condition $\Delta(\mathscr{A})$. A generalization of Proposition 4.2 to the setting of $\$ 3$ would also be useful.

A question related to Theorem 3.2 is the following. If the operators $\left\{T_{n}\right\}$ are taken to be compact, then the resulting $K$ is also compact. It is possible that this $K$ is a best compact approximant to $T$ ? 
In [3] Axler, Berg, Jewell, and Shields employ what they call the "Basic Inequality" for a Banach space $X$. This inequality is similar to condition $\Delta(\mathscr{A})$ for $\mathscr{A}=\{0\}$, the zero operator. They show that the Basic Inequality is satisfied for $X=l^{p}, 1<p<\infty$. They also prove that the closed unit ball of $L^{\infty} / H^{\infty}+C$ has no extreme points. Two questions which arise are whether $\Delta(\mathscr{A})$ holds when $\mathscr{A}$ is the algebra of operators on $l^{p}(1<p<\infty)$ with upper triangular matrix representations with respect to the standard basis, and whether the closed unit ball of $\mathscr{L}(H) / \mathscr{A}+\mathscr{K}(H)$ has any extreme points if $\mathscr{A}$ is a nest algebra satisfying condition $\Delta(\mathscr{A})$.

Another line of questioning is related to the theory of $M$-ideals, introduced in 1972 by Alfsen and Effros [1]. Luecking [8] showed that $H^{\infty}+C / H^{\infty}$ is an $M$-ideal in $L^{\infty} / H^{\infty}$, and it seems reasonable to ask if $\mathscr{A}+\mathscr{K}(H) / \mathscr{A}$ is an $M$-ideal in $\mathscr{L}(H) / \mathscr{A}$ for any nest algebra $\mathscr{A}$. An affirmative answer would imply, by a result of Holmes, Scranton, and Ward [7], that the collection $\mathscr{S}_{T}=\{A+\mathscr{A} \in \mathscr{A}+\mathscr{K}(H) / \mathscr{A}$ : $d(T-A, \mathscr{A})=d(T, \mathscr{A}+\mathscr{K}(H))\}$ would algebraically span $\mathscr{A}+\mathscr{K}(H) / \mathscr{A}$ for each $T \in \mathscr{L}(H) \backslash \mathscr{A}+\mathscr{K}(H)$.

\section{REFERENCES}

1. Eric M. Alfsen and Edward G. Effros, Structure in real Banach spaces, Ann. of Math. (2) 96 (1972), 98-173.

2. William Arveson, Interpolation problems in nest algebras, J. Funct. Anal. 20 (1975), 208-233.

3. Sheldon Axler, I. David Berg, Nicholas Jewell and Allen Shields, Approximation by compact operators and the space $H^{\infty}+C$, Ann. of Math. (2) 109 (1979), 601-612.

4. T. Fall, W. Arveson and P. Muhly, Perturbations of nest algebras, J. Operator Theory 1 (1979), $137-150$.

5. P. R. Halmos, Quasitriangular operators, Acta Sci. Math (Szeged) 29 (1968), 283-293.

6. Richard B. Holmes and Bernard R. Kripke, Best approximation by compact operators, Indiana Univ. Math. J. 21 (1971), 255-263.

7. Richard Holmes, Bruce Scranton and Joseph Ward, Approximation from the space of compact operators and other M-ideals, Duke Math. J. 42 (1975), 259-269.

8. Daniel H. Luecking, The compact Hankel operators form an M-ideal in the space of all Hankel operators, Proc. Amer. Math. Soc. 79 (1980), 222-224.

9. J. R. Ringrose, On some algebras of operators, Proc. London Math. Soc. 15 (1965), 61-83.

Department of Mathematics, Bucknell University, Lewisburg, Pennsylvania 17837 